\title{
Research on Communication Resource Management System based on Metamaterials
}

\author{
Zhou Rong ${ }^{1, *}$ \\ ${ }^{1}$ School of Information Engineering, Wuhan University of Technology, Wuhan, China, 430074 \\ * Corresponding author: 125850438@qq.com
}

\begin{abstract}
Electromagnetic Metamaterial (EMM), which cannot be found in nature and possesses the novel electromagnetic properties of negative refraction, reversals of both Doppler shift and Cherenkov radiation, is a kind of artificial composed material. In order to solve the problem of long delay and high packet loss rate, the paper proposed a communication resource management system based on Metamaterials. By using the electromagnetic simulation method, the triple band and tunable transmission/reflection characteristics are investigated. Experiment result shows that the proposed method can improve the performance in the communication resource management.
\end{abstract}

\section{Introduction}

Electromagnetic Metamaterial (EMM), which cannot be found in nature and possesses the novel electromagnetic properties, e.g. negative refraction, reversals of both Doppler shift and Cherenkov radiation, is a kind of artificial composed material. It has very important potential applications in the physical areas of including electromagnetics, optics, and materials, and also the engineering areas of such as wireless communications and electronic engineering. Recently, it has excited highly research interests in the world, since the first experimental realization of such EMM in 2000. The realization methods of such EMM developed in recent years include the transmission-line structure, metallic resonance structure, and full dielectric structure. Also, the Photonic Crystals can also lead to some novel electromagnetic properties as negative refraction, and therefore it can be considered as a kind of EMM. Among the above mentioned design methods, the metallic resonance structure has huge research merit due to the advantages such as simple structure, easy fabrication method, and flexibility to construct 3D realization. However, for the metallic resonance EMM, the major drawback is that the operating bandwidth is fixed and very narrow.

Software engineering data refers to the data generated in the whole life cycle of software, including source codes, specification documents, and bug reports and so on. In most situations, software engineering data is the only information source for developers. With the rapid enlargement of software scale, to acquire manually the relevant information of software development and maintenance is becoming more and more difficult. Data mining technology can help to discover useful information from software engineering data automatically, which thus speeds up the process of software development.

Wu's [1] paper investigates some data mining problems in software engineering data. Identifier names play an important role in software maintenance. A good method name explicitly indicates the function of the method to the developers, while bad names mislead them. However, except for developers' individual experiences and some naming rules, the developers lack effective tools to guide them to name methods. We consider the problem of automatically choosing proper action names, or verbs, for methods as a classification problem. We build an automated method name based on text categorization. Experiments show that our approach can achieve up to $70 \%$ naming accuracy for Java methods.

In Fu's [2] paper, process neural networks is introduced into time series data mining to deeply studying on clustering, classification and prediction problems, combining with wavelet multi-resolution analysis and related technologies. A time series clustering method is proposed base on wavelet and improved self-organization process neural network for time series clustering problem. Original time series data is decomposed by wavelet. Under the principle of reserving clustering characteristics, the signal is reconstructed. And then reconstructed signal which has been fitted into time-varying functions is used as the input of process neural network. Self-organization PNN is trained by improved competition algorithm [3]. Making use of time-varying input characteristic of PNN, the timing signal characteristics processed by wavelet is considered adequately in clustering analysis. Network extracts implicit process 
mode characteristics of input function to self-organize. The improved competition learning algorithm is given. Clustering result of UCI datasets shows that the proposed approach can be applied to timing clustering effectively.

\section{The Framework of Dynamic Voltage Restorer}

In contrast to the rapid development of software industry, especially open source projects, we lack effective and efficient plagiarism detection tools to protect them from piracy. A less self-disciplined developer, who committed software plagiarism, may elude punishment by seriously disguising the original open source projects. Existing Program Dependence Graph (PDG) based approaches can identify a few kinds of intentional disguises. However, they are rather slow and can be defeated by PDG affecting disguises. We develop a Type Dependence Graph (PDG) based plagiarism detection tool called TPLAG. TPLAG reduces the time complexity of graph based plagiarism detection from exponential to polynomial, and can see through several disguises that confuse existing tools.

Since hundreds of bugs are reported every day for large software projects such as Mozilla, bug triage is obviously a heavy burden for developers. Automated triage can relieve developers from such boring work. Existing work treats bug triage as a text categorization problem [4-5]. We use the Latent Dirichlet Allocation (LDA) model to reduce the dimension of documents, and then classify the bug reports to implement bug triage. Software maintenance highly depends on the information implied in bug reports. A bug report with little useful information is of poor quality and very likely to be ignored. We propose a topic model based approach to analyze bug report and quantify the information in bug reports, i.e., bug report quality. Understanding WHAT has gone wrong and WHEN did it happen with an open source software project can provide high-level and useful information for software maintenance. We try to acquire such information from the bug repository of the project. The LDA model is applied to answer the WHAT question, and an event detection tool is developed to answer the WHEN question. Experiments validate the proposed methods in Chen's [6] paper. Figure 2 shows the basic procedure of data mining technology.

Figure 1 shows the composition of data mining technology.

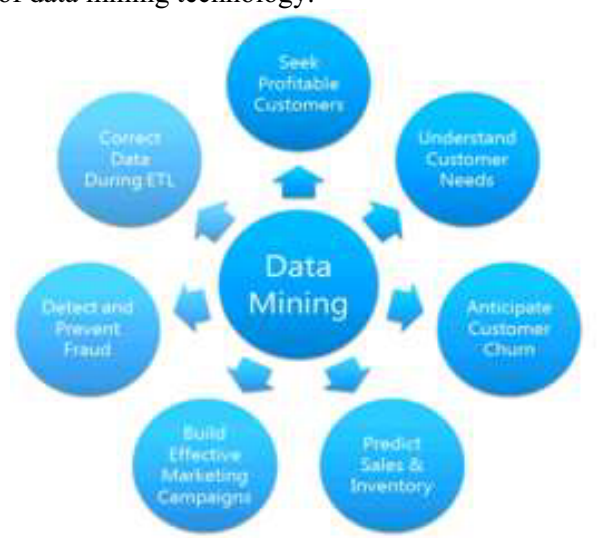

Fig. 1. The composition of data mining technology

Time series problem involves several fields, such as economy, meteorology, water conservancy, forestry etc. At present, time series data mining has been the focus of data mining, which has strong theoretical and practical significance. Because of some complex characteristics of time series data, for example, time variation, high dimension, noise jamming and volatility, time series data mining is always one of the difficulties in data mining research. Process neural network (PNN) is a development of traditional neural network in the time domain.

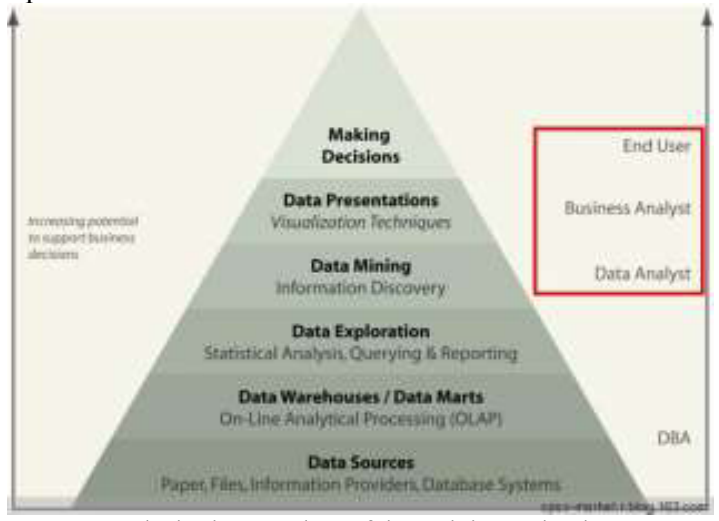

Fig. 2. The basic procedure of data mining technology 


\section{The Algorithm}

A less self-disciplined developer, who committed software plagiarism, may elude punishment by seriously disguising the original open source projects.

The basic model for online error identification as follows:

$$
\begin{gathered}
T S P(t)= \begin{cases}T S P_{1} & 0 \leq t \leq \Delta t \\
T S P_{2} & \Delta t \leq t \leq 2 \Delta t \\
\cdots & \\
T S P_{n} & (n-1) \Delta t \leq t \leq n \Delta t\end{cases} \\
\min d(T)=\sum_{k=1}^{n} \sum_{i=1}^{N} \sum_{j=1}^{N} c_{i, j}(k \square \Delta t) \\
\text { s.t } \quad \Delta t=\frac{T}{n}, \frac{\Delta c_{i, j}}{\Delta t}=0
\end{gathered}
$$

We may get the calculating method for the main index in the following equation (4)-(5):

$$
\begin{gathered}
M_{i j}=\exp \left(-\frac{\left\|x_{i}-x_{j}\right\|^{2}}{\sigma^{2}}\right) \\
L=\left[\begin{array}{lll}
L_{1} & & \\
& \ddots & \\
& & L_{k}
\end{array}\right]
\end{gathered}
$$

Their matching eigenvectors matrix is shown in the following equation (6):

$$
H=\left[h_{1}, h_{2}, \ldots, h_{k}\right]=A^{1 / 2} E
$$

So, we can get:

$$
\begin{gathered}
U_{i j}=\frac{H_{i j}}{\sqrt{\sum_{t=1}^{k} H_{i t}^{2}}}, i=1, \ldots, n, j=1, \ldots, k \\
P=I-A^{-1 / 2} M A^{-1 / 2}
\end{gathered}
$$

According to the equation (6), the calculating formula can be obtained in equation (7)-(10).

$$
\begin{gathered}
g(x, \omega)=\frac{1}{(2 \pi)^{3}} \int g(k, \omega) \exp \left(-i \mathrm{k}_{\square} \mathrm{x}\right) d \mathrm{k} \\
g(k, \omega)=\left\|\begin{array}{cr}
G_{i k}(k, \omega) & \gamma_{i}(k, \omega) \\
\gamma_{k}^{T}(k, \omega) & g(k, \omega)
\end{array}\right\| \\
G_{i k}=\left(\Lambda_{i k}+\frac{1}{\lambda} h_{i} h_{k}^{T}\right)^{-1}, g=-\left(\lambda+h_{i}^{T} \Lambda_{i j}^{-1} h_{j}\right)^{-1}, \quad \gamma_{i}=\frac{1}{\lambda} h_{k}^{T} G_{k i},
\end{gathered}
$$

\section{Results and Discussion}

Process neural network (PNN) is a development of traditional neural network in the time domain. Process input of PNN relax synchronization instantaneous limit on inputs in traditional neural network models, and is more general artificial neural network model. It has its own unique advantages in dealing with time related problems. Figure 3 shows the result for communication resource management system on the data optimization. 


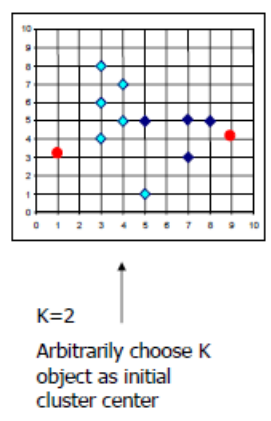

2013/11/26
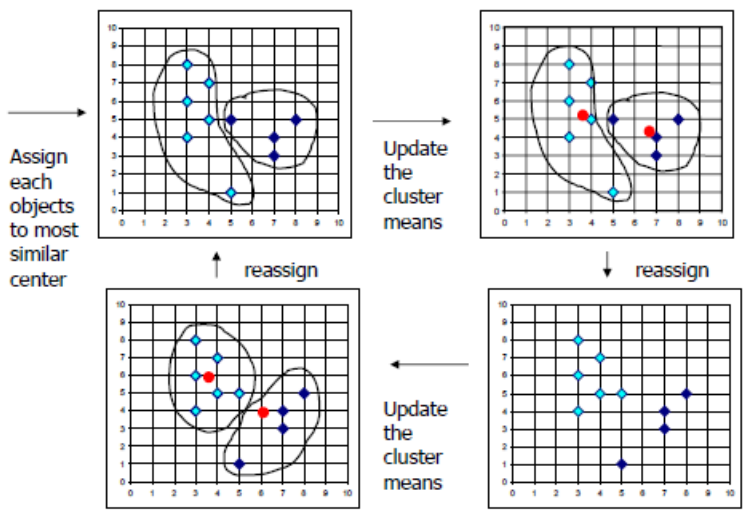

Fig. 3. The result for communication resource management system on the data optimization

This algorithm is defined as Valued K-means Genetic Algorithm. Based on the actual needs of the field of telecommunications knowledge discovery, the Data Mining Grid is an effective means of settlement to the problem that the efficiency of data mining algorithms will continue to fall when the data scale is increasing in geometric patterns. The improved competition learning algorithm is given. Clustering result of UCI datasets shows that the proposed approach can be applied to timing clustering effectively.

\section{References}

1. Wu He. Examining students' online interaction in a live video streaming environment using data mining and text mining. Computers in Human Behavior, (2013), pp. 291-304.

2. Fu Xiao, Cheng Fan. Data mining in building automation system for improving building operational performance. Energy \& Buildings, (2014), pp. 75-83.

3. Benrun Huang, Li Xia, Zhengguo Wu, Weiping Zhou. Control Strategy of A Dynamic Voltage Restorer (DVR) Based on Line Voltage Compensation. Energy Procedia, (2011), pp. 13-26.

4. Wei Dai, Mostafa Bassiouni. An improved task assignment scheme for Hadoop running in the clouds. Journal of Cloud Computing, (2013), pp. 21-33.

5. Wei Kuang Lai, Yi-Uan Chen, Tin-Yu Wu, Mohammad S. Obaidat. Towards a framework for large-scale multimedia data storage and processing on Hadoop platform. The Journal of Supercomputing, (2014), pp. $681-696$.

6. Zhijian Chen, Wenhai Luo, Dan Wu, Xiang Huang, Jian He, Yuanhuan Zheng, Di Wu. Exploiting application-level similarity to improve SSD cache performance in Hadoop. The Journal of Supercomputing, (2014), pp. 703-715. 